\title{
BRIEF COMMUNICATION \\ High resolution NanoSIMS imaging of deuterium distributions in 316 stainless steel specimens after fatigue testing in high pressure deuterium environment
}

\author{
Greg McMahon (iD) ${ }^{1}$, Bryan D. Miller ${ }^{2}$ and M. Grace Burke ${ }^{3}$
}

It is irrefutable that the presence of hydrogen reduces the mechanical performance of many metals and alloys used for structural components. Several mechanisms of hydrogen-assisted cracking (HAC) of steels have been postulated. The direct evidence of the mechanisms by which hydrogen embrittles these materials has remained elusive. This is by virtue of our difficulty to directly observe the hydrogen distribution at spatial resolutions less than $100 \mathrm{~nm}$ and analysis volumes greater than $1 \times 10^{9}$ atoms at microstructural features such as grain boundaries, dislocations, twins, stacking faults and sub-micron inclusions that are all potential hydrogen trapping sites postulated to be responsible for the degradation of mechanical performance. Here, we report on an experimental methodology combining an elaborate fatigue testing protocol in an enriched gaseous deuterium environment with NanoSIMS (secondary ion mass spectrometry) imaging for detection of deuterium at spatial resolutions as low as $100 \mathrm{~nm}$ and accompanying TEM analysis. Type 316 stainless steel compact tension specimens were precharged in deuterium followed by fatigue testing at high stress ratio $(0.7)$, low delta $\mathrm{K}(\sim 11 \mathrm{MPa} \sqrt{ } \mathrm{m})$, and a frequency of 1 cycle per minute using a sawtooth waveform with a rise time of $30 \mathrm{~s}$ in high pressure $(68.9 \mathrm{MPa})$ gaseous deuterium $(99.999 \%$ purity) environment at room temperature. High resolution NanoSIMS imaging was then used to measure the deuterium distribution at the tip of and in the wake of secondary and tertiary fatigue cracks as well as at MnS inclusions. The use of deuterium eliminates the difficulties of interpreting hydrogen measurements by SIMS relating to the ubiquitous presence of hydrogen in all high vacuum systems and guarantees that deuterium measured by the NanoSIMS must be attributed to the fatigue testing protocol. This methodology has allowed us to directly observe the distribution of hydrogen in dislocation tangles ahead and in the wake of fatigue crack tips and at the interface of MnS inclusions. The protocol provides an avenue by which the path and speed with which hydrogen proceeds along its embrittling course of action may be directly followed through modifications of the fatigue testing parameters and/or alloy type and allows a means to validate at least qualitatively recently published models of enhanced hydrogen transport by dislocations.

npj Materials Degradation (2018)2:2 ; doi:10.1038/s41529-017-0023-0

\section{INTRODUCTION}

Hydrogen has a longstanding reputation as a damaging element in a broad range of metals and alloys, especially in terms of inservice degradation of structural components. ${ }^{1-4}$ In particular, the contribution of hydrogen to the acceleration of fatigue failure is well-documented..$^{5-10}$ Several mechanisms of hydrogen-assisted cracking (HAC) of steels have been postulated, including the longstanding decohesion theory introduced by Troiano ${ }^{11,12}$ and Oriani, ${ }^{3,13}$ and the hydrogen-assisted deformation mechanism first proposed by Beachem ${ }^{14}$ and verified by Birnbaum et al. ${ }^{15-17}$ using in situ transmission electron microscopy (TEM) straining experiments in $\mathrm{H}_{2}$. Birnbaum and Sofronis ${ }^{18}$ further developed these concepts into the hydrogen-enhanced localized plasticity (HELP) mechanism. Alternatively, Lynch ${ }^{19,20}$ introduced the adsorption induced localized slip process (also known as adsorption induced dislocation emission), whereby adsorbed hydrogen weakens interatomic bonds facilitating dislocation nucleation at the crack tip. Dislocation injection on different crystallographic planes serves to advance the crack, while dislocation activity ahead of the crack tip can lead to voids which can then interact with the crack growing by the alternate slip mechanism to advance the crack front. More recently, another mechanism whereby hydrogen-induced deformation twins play a significant role in the acceleration of fatigue crack growth rates in the presence of hydrogen was suggested. ${ }^{8}$ The picture that emerges from the collective volume of research is that, when demonstrating and modeling the damaging effect of hydrogen, most studies only report the bulk concentration of hydrogen present in the test specimen measured by methods such as thermal desorption spectroscopy, ${ }^{21,22}$ or in the test atmosphere. Only a small volume of work is presented regarding the localized distribution of hydrogen around crack tips and other microstructural features such as grain boundaries, dislocations, twins, stacking faults, and inclusions. These include older studies with TEM replica-based tritium microautoradiography technique capable of spatial resolutions of $300 \mathrm{~nm}$ pioneered by Tiner and Co-workers ${ }^{23-25}$ and refined by Lacombe et al. at Universite Paris Sud ${ }^{26-28}$ and secondary ion mass spectrometry (SIMS) ion microscopy ${ }^{29,30}$ studies with lateral resolutions limited to $1 \mu \mathrm{m}$. Emerging more recently is work using atom probe tomography (APT), which has

\footnotetext{
${ }^{1}$ University of Manchester, School of Materials, Manchester M13 9PL, UK; ${ }^{2}$ Naval Nuclear Laboratory, West Mifflin, PA 15122, USA and ${ }^{3}$ University of Manchester, Materials Performance Centre, School of Materials, Manchester M13 9PL, UK

Correspondence: Greg McMahon (gregmcmahon345@gmail.com)
}

Received: 10 March 2017 Revised: 10 November 2017 Accepted: 14 November 2017

Published online: 16 January 2018 
atomic resolution but samples a much smaller volume $(\sim 100,000$ $\mathrm{nm}^{3}$ compared to greater than $1 \times 10^{9} \mathrm{~nm}^{3}$ for NanoSIMS), ${ }^{31,32}$ as well as scanning probe methods ${ }^{33,34}$ that show promise for quantification but whose spatial resolution is of the order of hundreds of microns. NanoSIMS imaging bridges the gap between these two methods, providing a better spatial resolution than tritium autoradiography (and also dispensing with the need to work with radioactive materials) while simultaneously sampling larger volumes than APT, while also offering the potential for absolute quantification of hydrogen concentration using well characterized matrix-matched standards.

\section{RESULTS}

In this study, we directly show the distribution of hydrogen, in the form of deuterium, around primary and tertiary fatigue crack tips as well as MnS inclusions in two type $316 \mathrm{~L} / 316 \mathrm{LN}$ stainless-steel alloys. Both alloys are similar in composition (differing mainly in $\mathrm{S}$ and $\mathrm{N}$ content as shown in Table 1) and contain a microstructure consisting of large $(50-150 \mu \mathrm{m})$ equiaxed grains; however, the $316 \mathrm{~L}$ steel contains MnS particles due to it elevated level of $\mathrm{S}$ $(0.012 \mathrm{wt} \%)$. Prior to fatigue testing, compact tension specimens $(0.5 \mathrm{~T})$ where precharged with deuterium using the gas phase charging method. The precharging conditions $\left(300^{\circ} \mathrm{C} / 138 \mathrm{MPa}\right.$ for 124 days) insured that a concentration of $\sim 140 \mathrm{wppm}$ of deuterium was present (as calculated using relations). ${ }^{35}$ The measured fatigue crack growth rates, $3.84 \times 10^{-5} \mathrm{~mm} /$ cycle for heat AS192 and $1.99 \times$ $10^{-5} \mathrm{~mm}$ /cycle for heat AS198, were between $1.8 \times$ and $2.6 \times$ higher than those measured during comparable testing performed on uncharged material in ambient temperature air. The fatigue testing resulted in primarily transgranular cracking with a relatively uniform crack front. The fracture surfaces are faceted with indications of planar slip and "fissuring" which is suggestive of cracking on secondary slip planes.

Analysis of hydrogen in magnetic sector SIMS, though experimentally possible, is fraught with artefacts and thus difficult to interpret. The omnipresence of gaseous hydrogen-containing molecules even in the best vacuum systems invariably adsorb on the sample surface and are subsequently measured by the mass spectrometer. A large fraction of the hydrogen signal therefore originates from the vacuum system and not the sample. The best approach to alleviate this potential for artefact is to use deuterium in the experimental design. With a natural isotopic abundance of only $0.0115 \%$, the fraction of deuterium in the ultra high vacuum analysis chamber will be negligible. A second difficulty not unique to hydrogen but to all SIMS analysis is the issue of quantification. Secondary ion yields are known to vary over several orders of magnitude depending upon the material chemistry, and true quantification requires matrix-matched standards, which are often difficult to produce. However, relative differences in concentration can be measured by normalizing the signal of interest to a signal representing the matrix. In this work, we have used oxygen as a matrix signal as it has a high secondary ion yield, is an elemental ion so issues of molecular secondary ion formation are avoided, is distributed uniformly in the steel, and is also present in the cracks

Table 1. Composition (wt\%) of the two type 316 stainless steel alloys used in this study

\begin{tabular}{|c|c|c|c|c|c|c|c|c|c|}
\hline Material & C & $\mathrm{Mn}$ & Si & $\mathrm{P}$ & $S$ & $\mathrm{Cr}$ & Mo & $\mathrm{Ni}$ & $\mathrm{N}$ \\
\hline $\begin{array}{l}\text { 316L (Heat } \\
\text { AS192) }\end{array}$ & 0.016 & 1.77 & 0.58 & $<0.01$ & 0.012 & 16.8 & 2.3 & 11.2 & 0.059 \\
\hline $\begin{array}{l}316 \mathrm{LN} \\
\text { (Heat } \\
\text { AS198) }\end{array}$ & 0.016 & 1.10 & 0.45 & 0.017 & $<0.001$ & 17.3 & 2.6 & 12.1 & 0.140 \\
\hline
\end{tabular}

which aids in their delineation, especially at the fine crack tips, from the stainless steel matrix.

The images in Fig. 1 are the deuterium and oxygen isotopic maps (Fig. 1a, b). The oxygen image clearly shows the location of the crack. The derived deuterium/oxygen $(D / O)$ ratio image is displayed (Fig. 1c) using a hue-saturation-intensity (HSI) transformation, where the hue or color represents the actual ratio value, and the intensity is representative of the counting statistics in that same area. Thus, our eye is guided to areas of significant enrichment and away from those with poor counting statistics, which will be less statistically reliable. As a result of the high oxygen signal from the crack tip, the $\mathrm{D} / \mathrm{O}$ ratio is significantly lower in the crack itself, demonstrated by the blue hue in the HSI image, and thus clearly delineates the crack from the matrix stainless steel. Several regions of enhanced D/O ratios are noted by the hot spots in the yellow-pink hue range, in the wake of the crack and near the tips of both the primary and a tertiary crack. These regions indicate a $\mathrm{D} / \mathrm{O}$ ratio at least $50 \%$ higher than in the majority of other areas in the bulk of the sample. However, to completely eliminate any effect of objective biasing of the ratio image, Fig. 1d shows that D/O ratio image once again but with a set of regions of interest also drawn. These are a result of a segmentation process whereby the ratio image is analyzed for contiguous regions of a minimum area (16 pixels ${ }^{2}$ in this case, with a pixel size of $78 \mathrm{~nm}$ ) with a mean ratio value at least $50 \%$ higher than the background level measured in areas away from the crack tip in the same image. We note several of these regions of interest (ROIs) directly adjacent the crack tip, some along the wake of the crack, and a third cluster at the tip of a tertiary crack. The areas of these ROls range between approximately 0.12 and $0.34 \mu \mathrm{m}^{2}$ in size. Comparing Fig. 1c, d shows a slight difference in where the apparent enrichments are located, and demonstrates the importance of having a robust method to extract regions of enrichment that are not biased by visual perception but given by the actual measurement values.

A similar set of images is presented in Fig. 2, but from another crack tip at much higher spatial resolution. The HSI D/O image in Fig. 2c again directs our attention to two larger "hot spots", with almost twice the ratio values, adjacent and in front of the crack tip.

Following NanoSIMS analysis, the samples were lightly repolished to remove damage caused by the primary $\mathrm{Cs}^{+}$ion beam. TEM samples were prepared from the crack tip region using the focused ion beam lift out technique and examined using a $200 \mathrm{kV}$ accelerating voltage. The presence of a cellular structure (Fig. 3a), whose boundaries were determined to be formed by networks of dislocations (Fig. 3b) was observed. These images were acquired approximately $4-5 \mu \mathrm{m}$ ahead of the crack tip and show highly localized regions of dislocation tangles. The region examined is in fact quite similar to the area shown in Fig. 1, and where several clusters of enriched D/O ratio values are observed. Magnifying these regions to bring them to the same magnification as the TEM images, as in Fig. 3c, the cellular appearance of the image is strikingly similar to that of the TEM image in Fig. 3a, strongly suggesting that the dislocation tangles are strong trap sites for deuterium.

Along with imaging deuterium in the vicinity of crack tips, we have also used this imaging capability to examine the potential penetration of hydrogen in MnS inclusions. For this set of experiments the detectors were positioned to measure ${ }^{32} \mathrm{~S}^{-}$, ${ }^{32} \mathrm{~S}^{1} \mathrm{H}^{-}$, and ${ }^{32} \mathrm{~S}^{2} \mathrm{H}^{-}$. Other mass interferences based on the three isotopes of sulfur or molecular ions stemming from $\mathrm{O}_{2}$ are easily separated with the instrument. Typical images are presented in Fig. 4, showing the secondary electron image and the aforementioned isotopic images and the ${ }^{32} \mathrm{~S}^{2} \mathrm{H}^{-}{ }^{32} \mathrm{~S}^{-} \mathrm{HSI}$ ratio image. The enrichment around the $\mathrm{MnS}$ inclusion is striking, and the fact that the ${ }^{32} \mathrm{~S}^{1} \mathrm{H}^{-}$, and ${ }^{32} \mathrm{~S}^{2} \mathrm{H}^{-}$appear so different rules out any possible mass interference effect, as the ${ }^{33} \mathrm{~S}^{1} \mathrm{H}^{-}$image would look identical to the ${ }^{32} \mathrm{~S}^{1} \mathrm{H}^{-}$, but weaker in signal owing to the much lower natural abundance of ${ }^{33} \mathrm{~S}^{-}$. Although similar findings have been 

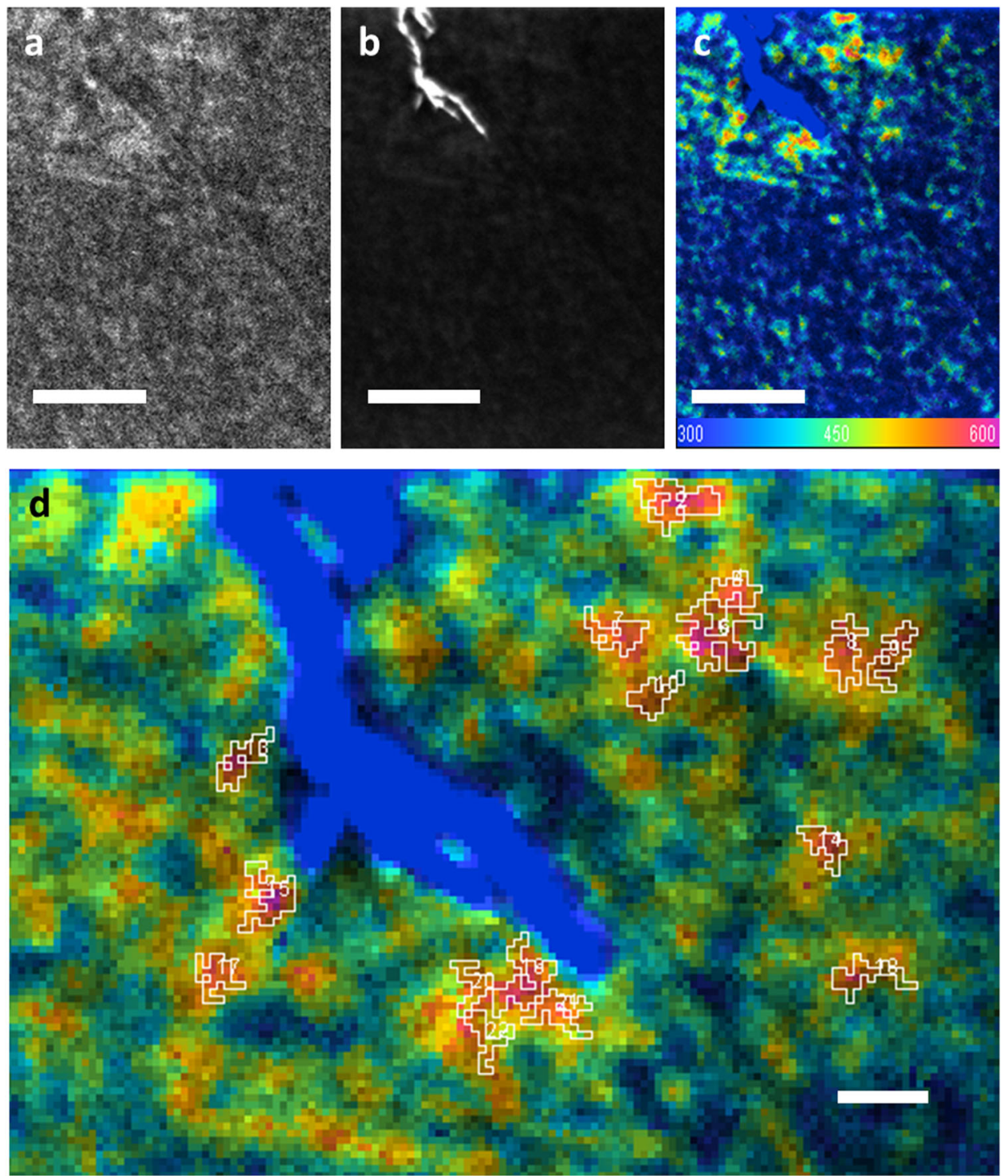

Fig. 1 NanoSIMS isotopic images and derived ratio images at crack tip from sample heat AS192. $\mathbf{a}{ }^{2} \mathrm{H}$ image $\mathbf{b}{ }^{16} \mathrm{O}$ image $\mathbf{c}{ }^{2} \mathrm{H} /{ }^{16} \mathrm{O}$ ratio displayed as HSI image. Scale bar in a-c is $5 \mu \mathrm{m} . \mathbf{d}^{2} \mathrm{H} /{ }^{16} \mathrm{O}$ ratio ratio showing locations of ROI's generated by segmentation algorithm showing contiguous regions with a minimum area of 16 pixels $^{2}$ (pixel size $=78 \mathrm{~nm}$ ) and a mean ratio at least $50 \%$ higher than the background taken from an area away from the crack tip. Scale bar is $1 \mu \mathrm{m}$

observed using tritium autoradiography in cathodically charged samples, $^{36-38}$ our data provide direct evidence that these inclusions trap hydrogen in gaseous environments as well. Further longer term exposure studies would be required on higher $S$ content alloys to observe if the idea proposed by Hanninen ${ }^{9,10}$ postulating that MnS inclusions act as hydrogen trap sites in low alloy steels and their subsequent dissolution in aqueous environments (as was recently directly observed through in situ TEM/EDS examination) ${ }^{39}$ changes crack tip electrochemistry and enhances hydrogen absorption also holds in gaseous environments.

\section{DISCUSSION}

The distribution of deuterium "hot spots" in localized regions within the cyclic plastic zone, as evident in Figs. 1 and 2, are potentially significant to how hydrogen may influence the deformation process and help elucidate which mechanism of $\mathrm{HAC}$ is responsible for the fatigue crack growth, at least under these test conditions. Fatigue loading is known to evolve complex deformation structures with locally high densities of dislocations, ${ }^{40-42}$ as are similarly observed in the present study (Fig. 3). The localization of hydrogen to these deformation structures, as is strongly suggested by Fig. 3, is consistent with hydrogen-enhanced dislocation nucleation and/or localization. As described by the HELP mechanism, ${ }^{18}$ hydrogen segregated to dislocations can reduce their interaction energy with other dislocations through modification of the elastic stress field. Therefore, the ability of dislocations to overcome obstacles will be enhanced and it directly follows that dislocation mobility will be increased at lower applied stress levels. Obstacles need not only be solute, but also can be other dislocations, and so a further manifestation of this effect is a smaller dislocation-dislocation distance in regions of high dislocation density, which can affect local stress states and potentially influence crack propagation. If this were indeed the case, we would also expect to see regions of higher hydrogen concentration resulting from the enhanced transport of hydrogen by dislocations which is clearly the case in Figs. 1-3. These results and method of analysis are of particular interest in light of the modeling of hydrogen transport by dislocations recently described by Dadfarnia et al. ${ }^{43}$ Although their model does not 

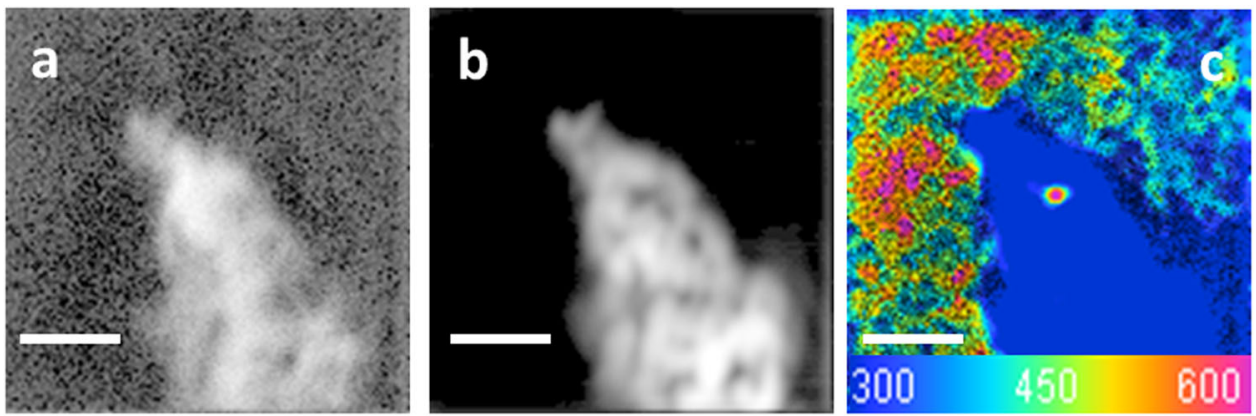

Fig. 2 Higher resolution NanoSIMS isotopic images and derived ratio images at crack tip from sample heat AS198. ${ }^{2} \mathrm{H}$ image $\mathbf{b}{ }^{16} \mathrm{O}$ image $\mathbf{c}$ ${ }^{2} \mathrm{H} /{ }^{16} \mathrm{O}$ ratio displayed as $\mathrm{HSI}$ image. Scale bar is $1 \mu \mathrm{m}$
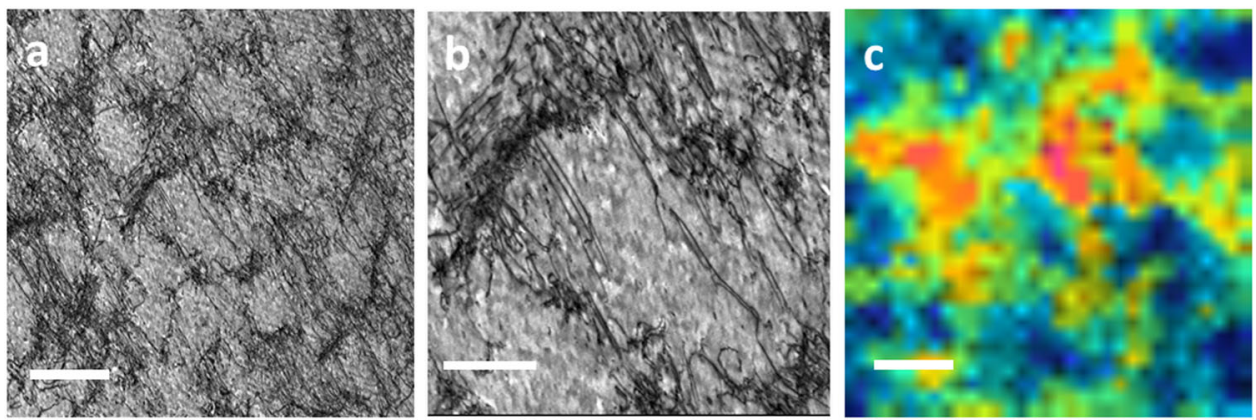

Fig. 3 Dislocation structure at crack tip from sample heat AS192. a TEM bright field image in crack wake region approximately 4-5 $\mu \mathrm{m}$ from crack. Scale bar is $500 \mathrm{~nm}$. b Same region imaged at higher magnification showing dislocations clusters are responsible for forming the cellular network observed in a. Scale bar is $200 \mathrm{~nm} . \mathrm{c}^{2} \mathrm{H} /{ }^{16} \mathrm{O}$ ratio displayed as HSI image in the crack wake region $4-5 \mu \mathrm{m}$ from crack, with the same magnification as in (a). Note areas of high and low deuterium also display cellular-like structure with cell sizes very similar to those observed in (a). Scale bar is $500 \mathrm{~nm}$
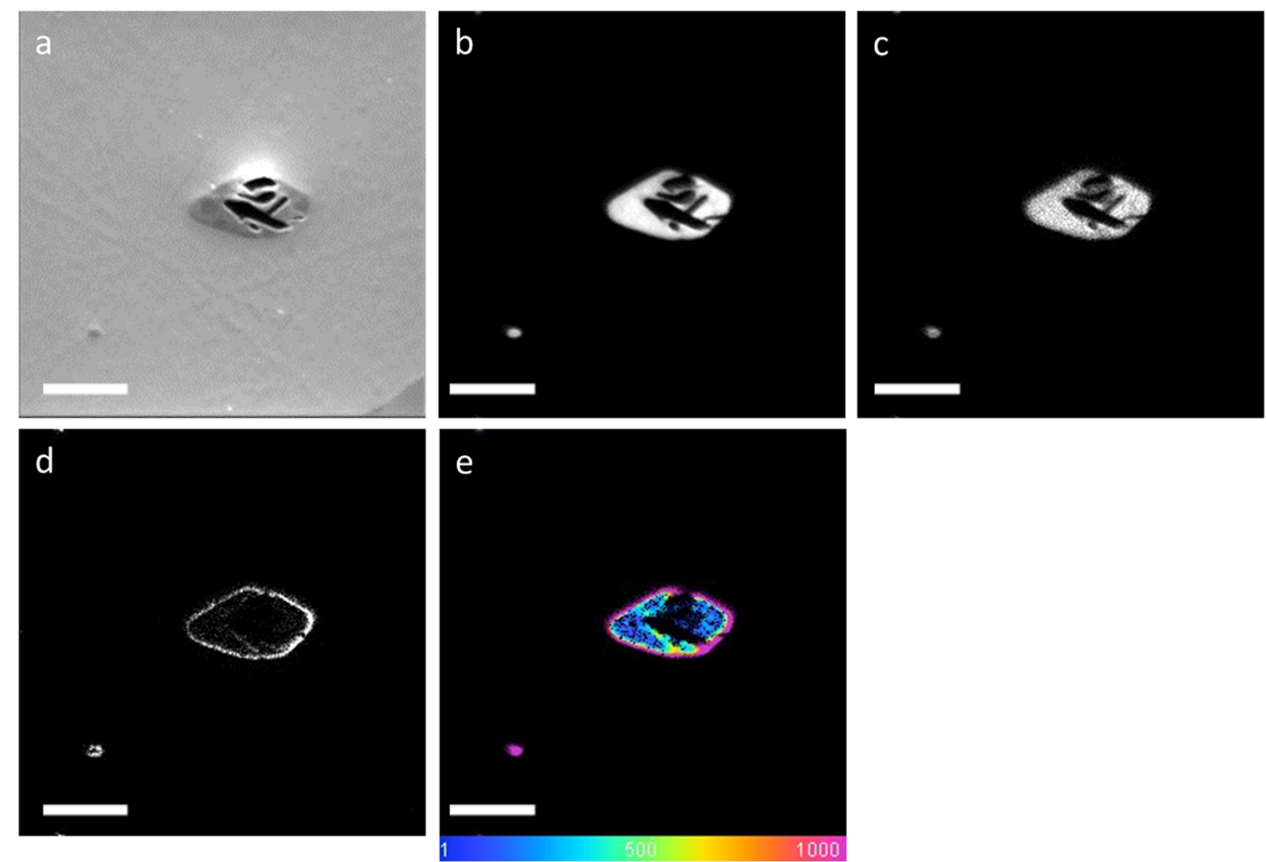

Fig. 4 NanoSIMS isotopic images and derived ratio images from MnS inclusion in sample heat AS192 (higher sulfur content) matrix away from crack tip. a Secondary electron image $b^{32} \mathrm{~S}^{-}$image $c^{32} \mathrm{~S}^{1} \mathrm{H}^{-}$image $\mathbf{d}^{32} \mathrm{~S}^{2} \mathrm{H}^{-}$image, and $\mathbf{e}^{32} \mathrm{~S}^{2} \mathrm{H}^{-} \beta^{32} \mathrm{~S}^{-}$ratio displayed as $\mathrm{HSI}$ image. Scale bar is $5 \mu \mathrm{m}$

describe a fatigue test, their predicted transport of hydrogen away from the crack tip as well as loss of pre-charged hydrogen from the crack tip site are in line with what is observed in this study. Thus, the methodology presented herein provides a means to experimentally validate these and future models, in response to one of the future research directions suggested in the recent review of advances on hydrogen embrittlement of structural materials. $^{44}$ 


\section{METHODS}

Samples for NanoSIMS analysis were prepared from the compact tension specimens using standard metallographic procedures with a final polish using an oxide polishing suspension. No etchant was used thereby avoiding sources of potential contamination. High spatial resolution SIMS images mapping the distribution of the elemental ${ }^{1} \mathrm{H}^{-},{ }^{2} \mathrm{H}^{-},{ }^{12} \mathrm{C}^{-},{ }^{16} \mathrm{O}^{-}$, and ${ }^{28} \mathrm{Si}^{-}$and the molecular ${ }^{16} \mathrm{O}^{2} \mathrm{H}^{-}$and ${ }^{32} \mathrm{~S}^{2} \mathrm{H}^{-}$secondary ions were acquired with a Cameca NanoSIMS 50L ion microprobe employing a $\mathrm{Cs}^{+}$primary ion beam. The beam with approximately a $100 \mathrm{~nm}$ spot size is rastered across the sample surface and the secondary ions produced by sputtering processes are filtered first for energy and subsequently for mass by a magnetic sector with a fixed magnetic field. Seven electron multiplier detectors are stationed at the appropriate radii to simultaneously detect and measure the secondary ions of interest from the same sputtered volume. By synchronizing the detection with the raster of the primary $\mathrm{Cs}^{+}$ beam, images are formed analogous to image acquisition in a scanning electron microscope. In fact, secondary electrons are also produced during the sputtering process and are used to produce a secondary electron image. Up to several hundred image acquisitions from the same area are acquired for each analysis, and the stack of images produced are aligned to a specific prominent feature in the image and then summed to create a single mass image with statistically relevant counts. More detailed description of the instrumentation can be found in Lechene et al. ${ }^{45}$ All data was analyzed with the open source OpenMIMS ${ }^{46}$ software plugin for ImageJ. ${ }^{47}$

\section{Data availability}

The NanoSIMS image data that support the findings are available from the corresponding author upon reasonable request.

\section{ACKNOWLEDGEMENTS}

The late William J. Mills is gratefully acknowledged for his assistance in planning the fatigue tests used to generate the specimens evaluated in this work. Fatigue tests were conducted by the Hydrogen Effects on Materials Laboratory (Brian Somerday, Ken Lee, and Jeff Campbell) at Sandia National Laboratory, Livermore, California. The authors thank Sibylle Schilling for metallographic sample preparation. R. Morris is gratefully acknowledged for preparing the FIB specimens for TEM analysis. Funding for the acquisition of the NanoSIMS by HEFCE (Higher Education Funding Council for England) from the UK Research Partnership Investment Fund (UKRPIF) Round 2 is gratefully acknowledged. Funding for the acquisition of the NanoSIMS by HEFCE (Higher Education Funding Council for England) from the UK Research Partnership Investment Fund (UKRPIF) Round 2 is gratefully acknowledged. Project funding from the Naval Nuclear Laboratory is also gratefully acknowledged.

\section{AUTHOR CONTRIBUTIONS}

B.M. planned and coordinated the fatigue testing, performed the TEM analysis of dislocation structures, and, with M.G.B., conceived the characterization work reported herein. G.M. performed all SIMS analysis and data processing. Data interpretation was discussed amongst all three authors led by G.M. All three authors contributed to writing the manuscript.

\section{ADDITIONAL INFORMATION}

Competing interests: The authors declare no competing financial interests.

Publisher's note: Springer Nature remains neutral with regard to jurisdictional claims in published maps and institutional affiliations.

\section{REFERENCES}

1. Cotterill, P. The hydrogen embrittlement of metals. Prog. Mater. Sci. 9, 205-301 (1961).

2. Bernstein, I. M. The role of hydrogen in the embrittlement of iron and steel. Mater. Sci. Eng. 6, 1-19 (1970).

3. Oriani, R. A. Hydrogen embrittlement of steels. Ann. Rev. Mater. Sci. 8, 327-357 (1978).

4. Hirth, J. P. Effects of hydrogen on the properties of iron and steel. Met. Trans. A 11, 861-890 (1980)

5. Murakami, Y., Kanezaki, T., Mine, Y. \& Matsuoka, S. Hydrogen embrittlement mechanism in fatigue of austenitic stainless steels. Metall. Mater. Trans. A 39A, 1327-1339 (2008).
6. Doig, P. \& Jones, G. T. A model for the initiation of hydrogen embrittlement cracking at notches in gaseous hydrogen environments. Metall. Mater. Trans. A. 8A, 1993-1998 (1977).

7. Martin, M. L., Sofronis, P., Robertson, I. M., Awane, T. \& Murakami, Y. A microstructural based understanding of hydrogen-enhanced fatigue of stainless steels. Int. J. Fatigue 57, 28-36 (2013).

8. Yamabe, J., Matsumoto, T., Matsuoka, S. \& Murakami, Y. A new mechanism in hydrogen-enhanced fatigue crack growth behaviour of a $1900 \mathrm{MPa}$ class highstrength steel. Int. J. Fract. 177, 141-162 (2012).

9. Hanninen, H., Torronen, K., Kemppainen, M. \& Salonen, S. On the mechanisms of environment sensitive cyclic crack growth of nuclear pressure vessel steels. Corros. Sci. 23, 663-679 (1983).

10. Hanninen, H., Cullen, W. \& Kemppainen, M. Effects of MnS inclusion dissolution on environmentally assisted cracking in low-alloy and carbon steels. Corrosion 46, 563-573 (1990).

11. Troiano, A. R. The role of hydrogen and other interstitials in the mechanical behaviour of metals. Trans. ASM 52, 54-80 (1960).

12. Scott, T. E. \& Troiano, A. R. Interstitials and fracture of metals. Nature 185, 372-373 (1960).

13. Oriani, R. A. Whitney award lecture-1987: hydrogen-the versatile embrittler. Corrosion 43, 390-397 (1987).

14. Beachem, C. D. A new model for hydrogen-assisted cracking (hydrogen "embrittlement"). Metall. Trans. 3, 437-451 (1972).

15. Matsumoto, T., Eastman, J. \& Birnbaum, H. K. Direct observations of enhanced dislocation mobility due to hydrogen. Scr. Metall. 15, 1033-1037 (1981).

16. Tabata, T. \& Birnbaum, H. K. Direct observations of hydrogen enhanced crack propagation in iron. Scr. Metall. 18, 231-236 (1984).

17. Robertson, I. M. \& Birnbaum, H. K. An HVEM study of hydrogen effects on the deformation and fracture of nickel. Acta. Metall. 34, 353-366 (1986).

18. Birnbaum, H. K. \& Sofronis, P. Hydrogen-enhanced localized plasticity-a mechanism for hydrogen-related fracture. Mater. Sci. Eng. A176, 191-202 (1994).

19. Lynch, S. P. Environmentally assisted cracking: overview of evidence for an adsorption-induced localised-slip process. Acta. Metall. 36, 2639-2661 (1988).

20. Lynch, S. P. Mechanisms and kinetics of environmentally assisted cracking: current status, issues and suggestions for further work. Metall. Mater. Trans. 44A 1209-1229 (2013).

21. Li, D., Gangloff, R. P. \& Scully, J. R. Hydrogen trap states in ultrahigh-strength AERMET 100 steel. Metall. Mater. Trans. A 35A, 849-864 (2004).

22. Suzuki, H. \& Takai, K. Summary of round-robin tests for standardizing hydrogen analysis procedures. ISIJ Int. 52, 174-180 (2012).

23. Tiner, N. A. \& Gilpin, C. B. Microprocesses in stress corrosion cracking of martensitic steels. Corrosion 22, 271-279 (1966).

24. Gilpin, C. B., Paul, D. H., Asunmaa, S. K. \& Tiner, N. A. Electron microautoradiography and its application to the study of hydrogen distribution in steel. ASTM-STP46415S Adv. Electron Metallogr. 6, 7-20 (1966).

25. Tiner, N. A., Mackay, T. L., Asunmaa, S. K. \& Ingersoll, R. G. Use of electron microautoradiography for evaluating microsegregation of hydrogen in titanium alloys. Trans. ASM 61, 195-202 (1968).

26. Aucouturier, M., Lapasset, G. \& Asaoka, T. Direct observation of hydrogen entrapment. Metallography 11, 5-21 (1978).

27. Asaoka, T., Lapasset, G., Aucouturier, M. \& Lacombe, P. Observation of hydrogen trapping in Fe- 0.15 wt\% $\mathrm{Ti}$ alloy by high resolution autoradiography. Corrosion 34, 39-47 (1978).

28. Paes de Oliveira, C., Aucouturier, M. \& Lacombe, P. Hydrogen trapping in BCC Fe$\mathrm{Cr}$ alloys (7-9.4 wt\% $\mathrm{Cr}$ ) as studied by microautoradiography-contribution of carbon-hydrogen interaction-consequences on hydrogen cracking. Corrosion 36, 53-59 (1980)

29. Kawamoto, K., Oda, Y., Noguchi, H., Fujii, H., Izumi, T. \& Itoh, G. Investigation of local hydrogen distribution around fatigue crack tip of a type 304 stainless steel with secondary ion mass spectrometry and hydrogen micro-print technique. $J$. Solid Mech. Mater. Eng. 3, 898-909 (2009).

30. Saintier, N., Awane, T., Olive, J. M., Matsuoka, S. \& Murakami, Y. Analyses of hydrogen distribution around fatigue crack on type 304 stainless steel using secondary ion mass spectrometry. Int. J. Hydrog. Energy 36, 8630-8640 (2011).

31. Takahashi, J., Kawakami, K. \& Tarui, T. Direct observation of hydrogen-trapping sites in vanadium carbide precipitation steel by atom probe tomography. Scr. Mater. 67, 213-216 (2012).

32. Chen, Y.-S. et al. Direct observations of individual hydrogen atoms at trapping sites in a ferritic steel. Science 355, 1196-1199 (2017).

33. Schaller, R. F., Thomas, S., Birbilis, N. \& Scully, J. R. Spatially resolved mapping of the relative concentration of dissolved hydrogen using the scanning electrochemical microscope. Electrochem. Commun. 51, 54-58 (2015).

34. Schaller, R. F. \& Scully, J. R. Spatial determination of diffusible hydrogen concentrations proximate to pits in a Fe-Cr-Ni-Mo steel using the scanning Kelvin probe. Electrochem. Commun. 63, 5-9 (2016). 
35. San Marchi, C., Somerday, B. P. \& Robinson, S. L. Permeability, solubility and diffusivity of hydrogen isotopes in stainless steels at high gas pressures. Int. J. Hydrog. Energy 32, 100-116 (2007)

36. Le, T. D. \& Wilde, B. E. An autoradiographic technique for studying the segregation of hydrogen absorbed into carbon and low alloy steels. Corrosion 39, 258-265 (1983).

37. Garet, M., Brass, A. M., Haut, C. \& Guttierez-Solana, F. Hydrogen trapping on non metallic inclusions in Cr-Mo low alloy steels. Corros. Sci. 40, 1073-1086 (1998).

38. Otsuka, T. \& Tanabe, T. Hydrogen diffusion and trapping process around MnS precipitates in alpha-Fe examined by tritium autoradiography. J. Alloys and Compounds 446-447, 655-659 (2007).

39. Schilling, S., Janssen, A., Zhong, X. L., Zaluzec, N. J. \& Burke, M. G. Liquid in situ analytical electron microscopy: examining SCC precursor events for type 304 stainless steel in H2O. Microsc. Microanal. 21, 1291 (2015).

40. Mughrabi, H., Ackermann, F. \& Herz, K. Persistant slip bands in fatigued facecentered and body-centered cubic metals. ASTM-STP35885S Fatigue Mech. 675 69-105 (1978).

41. Fielding, S. E. \& Stobbs, W. M. Dislocation structures in fatigued polycrystalline stainless steel. J. Microsc. 130, 279-288 (1983).

42. Gerland, M., Mendez, J., Violan, P. \& Ait Saadi, B. Evolution of dislocation structures and cyclic behaviour of a 316 L-type austenitic stainless steel cycled in vacuo at room temperature. Mater. Sci. Eng. A. 118, 83-95 (1989).

43. Dadfarnia, M. et al. Modeling hydrogen transport by dislocations. J. Mech. Phys. Solids 78, 511-525 (2015).
44. Dadfarnia, M. et al. Recent advances on hydrogen embrittlement of structural materials. Int. J. Fract. 196, 223-243 (2015).

45. Lechene, $C$. et al. High-resolution quantitative imaging of mammalian and bacterial cells using stable isotope mass spectrometry. J. Biol. 5, 20 (2006).

46. Lechene, C. et al. Center for Nanoimaging-Brigham and Women's Hospital. http:// nano.bwh.harvard.edu/openmims (2016).

47. Rasband, W. S., ImageJ, U. S. National Institutes of Health. Bethesda, Maryland. http://imagej.nih.gov/ij/ (1997-2016).

Open Access This article is licensed under a Creative Commons Attribution 4.0 International License, which permits use, sharing, adaptation, distribution and reproduction in any medium or format, as long as you give appropriate credit to the original author(s) and the source, provide a link to the Creative Commons license, and indicate if changes were made. The images or other third party material in this article are included in the article's Creative Commons license, unless indicated otherwise in a credit line to the material. If material is not included in the article's Creative Commons license and your intended use is not permitted by statutory regulation or exceeds the permitted use, you will need to obtain permission directly from the copyright holder. To view a copy of this license, visit http://creativecommons. org/licenses/by/4.0/.

(c) The Author(s) 2018 